\title{
TEOR DE MACRO E MICRONUTRIENTES E POTENCIAL FISIOLÓGICO DE SEMENTES EM GENÓTIPOS CRIOULO E MELHORADO DE FEIJÃO
}

\author{
Carla Xavier Alves ${ }^{1}$, Gilberto Antonio Peripolli Bevilaqua ${ }^{2}$, Luis Osmar Braga Schuch ${ }^{1}$, Irajá Ferreira Antunes ${ }^{2}$, \\ Paulo Eduardo Rocha Eberhardt ${ }^{1}$ \\ ${ }^{1}$ Universidade Federal de Pelotas - UFPel, Faculdade de Agronomia Eiseu Maciel( FAEM), Capão do Leão, RS. ${ }^{2}$ Embrapa \\ Clima Temperado, Pelotas, RS.
}

\section{RESUMO}

O feijão é cultivado principalmente por agricultores familiares, que geralmente utilizam sementes de anos anteriores, selecionadas pelos próprios agricultores, caracterizando-as como sementes crioulas, apresentando alto potencial para o uso direto pelos agricultores e utilização em programas de melhoramento. O trabalho teve o objetivo de avaliar a qualidade fisiológica e o teor de nutrientes em sementes de cultivares crioulas e melhoradas por seleção participativa, e a influência do teor desses nutrientes no potencial fisiológico das sementes. Foram utilizadas sementes provenientes dos ensaios de avaliação de cultivares conduzidos em São Luiz Gonzaga e Sobradinho, RS, da safra 2012. Foram avaliadas nas sementes os teores de: fósforo, cálcio, potássio, ferro, magnésio, zinco e manganês. Também foram avaliados a qualidade fisiológica por meio de: germinação, envelhecimento acelerado, teste de frio, condutividade elétrica e comprimento de raiz e parte aérea nas plântulas. Concluiu-se que as sementes das cultivares crioulas e melhoradas por seleção participativa apresentam elevado potencial fisiológico. Os genótipos AM-10, AS-7, Preto Ibérico TB 02-21 e ZL-1 destacaram-se pelo maior potencial fisiológico das sementes. Existe interação entre genótipo e ambiente para teor de nutrientes nas sementes. 0 teor de nutrientes exerce influência na qualidade das sementes, principalmente fósforo, cálcio, ferro e manganês.

Palavras-chave: Phaseolus vulgaris; interação genótipo x ambiente; vigor de sementes; composição química; correlação.

\section{MACRO AND MICRONUTRIENTS LEVELS AND PHYSIOLOGICAL POTENTIAL IN SEEDS OF LANDRACES AND BREEDING BEAN GENOTYPES}

\begin{abstract}
Bean is cultivated mainly by family farmers, who usually use seeds from previous years, selected by the farmers themselves, characterizing them as creole seeds, presenting high potential for direct use by farmers and use in breeding programs. The objective of this work was to evaluate the physiological quality and nutrient content of seeds of cultivar cultivars and improved by participatory selection and the influence of the content of these nutrients on the physiological potential of the seeds. Seeds from the trials of cultivars conducted in São Luiz Gonzaga and Sobradinho, RS, from the 2012 harvest were used. The seeds, phosphorus, calcium, potassium, iron, magnesium, zinc and manganese were evaluated in the seeds. The physiological quality was also evaluated by means of: germination, accelerated aging, cold test, electrical conductivity, root, and shoot length in the seedlings. It was concluded that the seeds of the cultivar creole and improved by participatory selection have high physiological potential. The genotypes AM-10, AS-7, Iberico Preto TB 02-21 and ZL-1 were distinguished by the higher physiological potential of the seeds. There is interaction between genotype and environment for nutrient content in seeds. The nutrient content exerts influence on the quality of the seeds, mainly phosphorus, calcium, iron and manganese.
\end{abstract}

Keywords: Phaseolus vulgaris; genotype ambient interaction; seed quality; nutrients; correlation. 


\section{INTRODUÇÃO}

O cultivo de feijão no Brasil ocorre praticamente durante todo o ano, distribuído por todo o território nacional e preferencialmente em unidades familiares (COSTA; VIEIRA, 2000). De acordo com Maeda e Mendonça (1990), o cultivo em várias épocas do ano deve-se ao fato do feijão não apresentar sensibilidade ao fotoperíodo. A cultura é suscetível a variações climáticas, como temperaturas do ar muito baixa ou alta, durante o estádio reprodutivo, e chuvas, principalmente na colheita, elementos climáticos que influenciam na escolha das melhores regiões e definem as épocas de semeadura mais adequadas (STONE; SARTORATO, 1994).

A semente utilizada no cultivo advém, na maior parte, de anos anteriores, a qual normalmente é selecionada pelo próprio agricultor por muitos anos, o que é caracterizado como cultivar crioula. Segundo Antunes et al. (2007) a espécie, por ser originária da América, possui grande variação quanto a características da planta e da semente como: forma, cor, tamanho e brilho. Conforme Pereira et al. (2009, 2011), a análise mais apurada destas variedades mostrou que genótipos crioulos podem apresentar características morfo-agronômicas e qualidade nutricional dos grãos destacadas. Além disso, as cultivares crioulas possuem um comportamento mais estável quanto à produtividade, porém com potencial de rendimento menor que as cultivares melhoradas e híbridas, e produzem relativamente bem em anos e condições climáticas desfavoráveis. As cultivares crioulas podem apresentar elevado potencial para tolerância a condições adversas, ao ataque de pragas e elevada qualidade de semente e representam perspectivas para uso direto dos agricultores, pois respondem ao interesse do consumidor, na medida em que existe a preocupação em incorporar essa diversidade nos sistemas de cultivo e na alimentação. Os cultivos desses genótipos pelos agricultores familiares também contribuem na conservação desses recursos genéticos (COELHO, 2010).
Ribeiro et al. (2008), avaliando cultivares crioulas de feijão, mostrou que algumas delas são promissoras por apresentarem alto potencial de rendimento de grãos e caracteres agronômicos desejáveis. Em Santa Catarina, Coelho et al. (2010), avaliando genótipos crioulos de feijão, concluíram que os mesmos apresentaram elevado potencial fisiológico em relação a testemunha, em função de maior germinação, comprimento da raiz primária, baixos valores de condutividade e elevada emergência em campo. As sementes, semelhante aos demais órgãos das plantas, apresentam composição química bastante variável por ser um órgão que se forma no final do ciclo da planta. Um genótipo que apresente maior capacidade em translocar e armazenar nutrientes na semente tem maior potencial em produzir sementes com elevado poder germinativo e vigor de plântulas mesmo sob condições adversas de estresse bióticos e abióticos (MARCOS FILHO, 2005).

Em várias funções nas plantas Fe e Zn são determinantes ou integrantes de diversos processos, tais como: síntese de proteínas, permeabilidade de membranas, absorção iônica, respiração, síntese de amido e controle hormonal (TEIXEIRA et al., 2005). Assim, constata-se a hipótese de que nutrientes estejam envolvidos na qualidade fisiológica das sementes. Com base no exposto o presente trabalho teve por objetivo: determinar os teores dos principais macro e micronutrientes e o potencial fisiológico das sementes, em genótipos de feijão crioulos e melhorados por seleção participativa produzidas em dois locais no Rio Grande do Sul e relacionar a influência dos principais nutrientes sobre a qualidade fisiológica das sementes.

\section{MATERIAL E MÉTODOS}

Foram utilizados oito genótipos crioulos, quatro genótipos do melhoramento participativo e duas testemunhas uma para grão de cor e um preto, provenientes de ensaio de avaliação de cultivares, cujas informações estão na Tabela 1. 
Tabela 1. Genótipos avaliados e suas características quanto à origem e cor do grão

\begin{tabular}{lcc}
\hline Genótipos & Origem & Cor do tegumento \\
\hline AM-10 & Seleção participativa & Preto \\
Amarelinho lolanda & Crioula & Amarelo \\
AS-7 & Seleção participativa & Preto \\
CK-4 & Seleção participativa & Preto \\
Guabiju Brilhante & Crioula & Preto \\
Preto lbérico & Crioula & Preto \\
TB 02-20 & Crioula & Preto \\
TB 02-21 & Seleção participativa & Preto \\
TB 02-25 & Crioula & Preto \\
TB 02-26 & Crioula & Vermelho \\
Vinho 141 & Crioula & Vinho \\
ZL-1 & Seleção participativa & Preto \\
\hline BRS Intrépido & Testemunha & Preto \\
Carioca & Testemunha & Bege \\
\hline
\end{tabular}

O experimento foi conduzido na safra de 2012 nos municípios de Sobradinho, com semeadura em 19 de outubro de 2011 e colheita em 22 de janeiro de 2012 e São Luiz Gonzaga, com semeadura em 17 de fevereiro de 2012 e colheita em 19 de maio de 2012, no Estado do Rio Grande do Sul. A adubação constituiu-se na aplicação de $300 \mathrm{~kg}$ há-1 da fórmula NPK 10-30-10, como padrão dos ensaios de feijão. As análises do solo foram realizadas dentro do planejamento da propriedade aonde foram instalados os experimentos e representam valores das áreas homogêneas maiores dentro da propriedade. $\mathrm{O}$ delineamento experimental foi de blocos casualizados com três repetições, sendo as parcelas compostas por quatro linhas de $4 \mathrm{~m}$ de comprimento e espaçadas de $0,5 \mathrm{~m}$. Por ocasião da maturação as plantas foram colhidas e trilhadas manualmente, e secas ao sol até umidade de aproximadamente $12 \%$.

Quanto aos ambientes de cultivo, em São Luiz Gonzaga, representa o planalto riograndense com solos de alta fertilidade típicos de basalto e altitude média de $231 \mathrm{~m}$. O solo utilizado foi um latossolo vermelho com plantio direto consolidado, sob cultivo anterior de milho. A área possui boa fertilidade com $37 \mathrm{mg}$ kg de potássio, $202 \mathrm{mg} \mathrm{kg}$ de fósforo e 2,5\% de matéria orgânica. O clima, segundo Koeppen, e Cfa com temperatura anual média de $190 \mathrm{C}$ e precipitação anual média de $1750 \mathrm{~mm}$, bem distribuída. Em Sobradinho, foi utilizado um solo de média fertilidade, formados a partir de basalto $e$ arenitos. A temperatura média anual é $18,6^{\circ} \mathrm{C}$ e com precipitação de $1476 \mathrm{~mm}$ de chuvas bem distribuídas. O solo utilizado foi um argissolo, com $24 \%$ de argila, $40 \mathrm{mg}$ kg de $\mathrm{P}, 120 \mathrm{mg} \mathrm{kg}$ de $\mathrm{K}$ e 1,6\% de matéria orgânica, sob cultivo convencional. Em ambos os locais o $\mathrm{pH}$ do solo estava próximo de 6, sendo o ideal para feijão.

Quanto as condições climáticas do ano em questão, observou-se em fevereiro e março temperaturas acima do normal e precipitação normal; abril, precipitação abaixo do normal e temperatura normal e maio precipitação abaixo do normal e temperatura acima do normal. Resumidamente pode-se dizer que a precipitação no início do desenvolvimento da cultura foi normal e ao final do ciclo houve baixas precipitações, porém em São Luiz Gonzaga, as precipitações ao final do ciclo foram maiores e durante a maior parte do ciclo as temperaturas estiveram acima do normal. As precipitações no Rio Grande do Sul ficaram abaixo do padrão climatológico em praticamente todo o Rio Grande do Sul, apenas em parte da campanha na região de Santana do Livramento, ficaram dentro do padrão devido a fortes chuvas ocorridas nos dias 17 e 18. As temperaturas mínimas ficaram pouco abaixo do padrão climatológico em grande parte do Estado. As temperaturas máximas ficaram pouco acima do padrão climatológico em todo o Rio Grande do Sul (DISME/INMET; CPPMet/UFPEL, 2011). No mês de dezembro, as precipitações no Rio Grande do Sul ficaram abaixo do padrão climatológico em grande parte do Estado, apenas no extremo oeste e nordeste (São Luiz Gonzaga), ficaram acima do padrão devido a chuvas intensas em curto período, onde o total de chuvas do mês ficou concentrado entre os dias 23 e 25 . As temperaturas mínimas ficaram abaixo do padrão climatológico em grande parte do Estado. As temperaturas máximas ficaram dentro do padrão climatológico no centro e 
noroeste do estado (DISME/INMET; CPPMet/UFPEL, 2012).

A qualidade fisiológica das sementes foi avaliada pelos seguintes testes: a) teste de germinação: foi realizado de acordo com as Regras para Análise de Semente (BRASIL, 2009), exceto o número de sementes que foi utilizado quatro repetições de 50 sementes; b) teste de frio: foi utilizado quatro repetições de 50 sementes, em rolo de papel germitest umedecido com água destilada. Os rolos de papel, acondicionados em sacos plásticos, foram mantidos à temperatura de $10^{\circ} \mathrm{C}$ por período de sete dias. Depois desse período os rolos passaram para germinador sendo o teste conduzido conforme descrito para o teste de germinação e a leitura final realizada após 5 dias; c) envelhecimento acelerado: seguindo a metodologia descrita por Marcos Filho (2005) e para o teste foram utilizadas quatro repetições de 50 sementes, dispostas em caixas plásticas do tipo gerbox adaptadas e colocadas em BOD regulada à $42^{\circ} \mathrm{C}$ permanecendo por $72 \mathrm{hs}$ e após realizado o teste de germinação e a leitura realizada aos cinco dias; d) condutividade elétrica: foram pesadas 25 sementes por repetição e colocadas em recipiente com água deionizada em temperatura de $25 \circ \mathrm{C}$ previamente estabilizada por 24 horas e após medida em condutivímetro. O valor obtido na leitura do condutivímetro foi dividido pelo peso da amostra e o resultado expresso em $\mu \mathrm{S} \mathrm{cm}^{-1} \mathrm{~g}^{-1}$; e) comprimento de raiz e de parte aérea de plântulas foi realizado em rolos de papel dispostos na $\mathrm{BOD}$ reguladas à $25^{\circ} \mathrm{C}$, com quatro repetições de 20 sementes cada, e a avaliação realizada aos cinco dias, medindo separadamente raiz e parte aérea.

Para análise do teor de nutrientes das sementes foram moídas aproximadamente $50 \mathrm{~g}$ de sementes, em um mini moinho, e acondicionadas em recipientes de vidro âmbar. Desta quantidade foram pesadas $0,25 \mathrm{~g}$ de cada amostra e em tubo plástico onde foi adicionado $1 \mathrm{ml}$ de peróxido de hidrogênio e $5 \mathrm{ml}$ de ácido nítrico para realização da digestão das mesmas.

As análises foram realizadas no laboratório da Central Analítica da Embrapa Clima Temperado, através da técnica de Espectrofotometria de absorção atômica -EAA. Foram determinados os teores de magnésio $(\mathrm{Mg})$ e cálcio (Ca) conforme metodologia de MIYAZAWA et al. (1992), manganês (Mn), (MIYAZAWA et al., 1992), ferro (Fe) e zinco (Zn)
(MALAVOLTA et al., 1989). Por Espectrofotometria com amarelo-de-vanadato foi determinado o teor fósforo (P) (MALAVOLTA et al., 1989) e através de Fotometria de chama o teor de potássio (K) (MIYAZAWA et al., 1992). As metodologias estão descritas em Silva (1999).

Os dados obtidos com os testes realizados foram submetidos a análise de variância e as médias agrupadas pelo teste de Scott-Knott a $5 \%$ de probabilidade para avaliação do potencial fisiológico e teor de nutrientes. Para analisar a influência do teor de nutrientes na qualidade das sementes, os dados foram submetidos a análise de correlação pelo coeficiente de Pearson, neste caso foram considerados como efetivos aqueles valores com coeficiente acima de 0,5.

\section{RESULTADOS E DISCUSSÃO}

Pode-se observar na Tabela 2 que houve diferença significativa entre os tratamentos bem como houve interação significativa entre genótipos e ambientes de cultivo para todas as variáveis analisadas, tanto de qualidade das sementes como os teores dos nutrientes.

O teor de $\mathrm{P}$, na semente, apresentou valor similar em Sobradinho e São Luiz Gonzaga, 4,40 e $4,63 \mathrm{~g} \mathrm{~kg}^{-1}$, respectivamente, e bastante próximos aos encontrados por Pereira et al. (2011), de 4,81 e 4,88 $\mathrm{g} \mathrm{kg}^{-1}$, também em genótipos crioulos em dois anos de avaliação (Tabela 2). Em Sobradinho, destacou-se o genótipo CK-4 que apresentou o teor mais alto, enquanto os teores mais baixos foram observados em TB 02-20, TB 02-21, TB 02-25 e TB 02-26. Entre as sementes produzidas em São Luiz Gonzaga, o melhor resultado foi com o genótipo AS-7 e o mais baixo Vinho 141.

Os teores mais altos de $\mathrm{K}$ foram observados em São Luiz Gonzaga, variando de 20,52 a 16,20 $\mathrm{g} \mathrm{kg}^{-1}$, com destaque para os genótipos AS -7, Preto Ibérico e Vinho 141, enquanto o menor resultado foi observado em CK-4. Em Sobradinho os valores médios foram menores e os melhores resultados foram observados em Amarelinho Iolanda, BRS Intrépido, Carioca e Vinho 141, variando de 15,21 a $14,20 \mathrm{~g} \mathrm{~kg}^{-1}$. Em São Luiz Gonzaga, TB 02-25 apresentou o resultado mais baixo de $11,29 \mathrm{~g} \mathrm{~kg}$ ${ }^{1}$. Os teores de potássio encontrados nos dois locais de cultivo foram superiores aos observados por Salum et al. (2008), entre 12,90 e 11,92 g Kg 1 , o que pode ser devido possivelmente pelo conteúdo do elemento no solo e características 
genéticas das cultivares. Entretanto são semelhantes aos resultados encontrados por
Pereira et al. (2011), que observaram valores entre 20,21 a $11,23 \mathrm{~g} \mathrm{Kg}^{-1}$.

Tabela 2. Teores de fósforo (P) e de potássio (K) em sementes provenientes de Sobradinho e São Luiz Gonzaga, RS, 2012.

\begin{tabular}{|c|c|c|c|c|}
\hline \multirow[t]{2}{*}{ Genótipos } & \multicolumn{2}{|c|}{$P\left(\mathrm{~g} \mathrm{~kg}^{-1}\right)$} & \multicolumn{2}{|c|}{$\mathrm{K}\left(\mathrm{g} \mathrm{kg}^{-1}\right)$} \\
\hline & Sobradinho & SL Gonzaga & Sobradinho & S L Gonzaga \\
\hline AM-10 & 4,12 B d & $5,71 \mathrm{Ab}$ & $12,56 \mathrm{~B} \mathrm{~b}$ & $18,92 \mathrm{Ab}$ \\
\hline Amarelinho Iolanda & $5,81 \mathrm{Ab}$ & 4,06 B b & $14,20 \mathrm{~B} \mathrm{a}$ & $17,98 \mathrm{AC}$ \\
\hline AS-7 & $4,23 \mathrm{~B} \mathrm{~d}$ & $7,55 \mathrm{~A} \mathrm{a}$ & 12,46 B b & $20,25 \mathrm{~A} \mathrm{a}$ \\
\hline BRS-Guerreiro $(T)^{*}$ & $5,64 \mathrm{Ab}$ & $4,81 \mathrm{~A} \mathrm{~b}$ & $15,21 \mathrm{~B} \mathrm{a}$ & $18,36 \mathrm{Ac}$ \\
\hline Carioca $(T)^{*}$ & $4,92 \mathrm{AC}$ & $5,05 \mathrm{Ab}$ & $14,92 \mathrm{~B} \mathrm{a}$ & $19,45 \mathrm{Ab}$ \\
\hline CK-4 & $7,20 \mathrm{Aa}$ & $4,75 \mathrm{~B} \mathrm{~b}$ & $12,89 \mathrm{~B} \mathrm{~b}$ & $16,20 \mathrm{Ad}$ \\
\hline Guabiju Brilhante & $4,25 \mathrm{Ad}$ & $3,04 \mathrm{~B} \mathrm{C}$ & $12,22 \mathrm{~B} \mathrm{~b}$ & $18,87 \mathrm{~A} \mathrm{~b}$ \\
\hline Preto Ibérico & $3,87 \mathrm{~B} \mathrm{~d}$ & $4,73 \mathrm{Ab}$ & $13,60 \mathrm{~B} \mathrm{~b}$ & $20,52 \mathrm{~A} \mathrm{a}$ \\
\hline TB 02-20 & $2,84 \mathrm{~B} \mathrm{e}$ & $5,52 \mathrm{Ab}$ & $12,89 \mathrm{~B} \mathrm{~b}$ & $19,39 \mathrm{~A} \mathrm{~b}$ \\
\hline TВ 02-21 & $3,29 \mathrm{~B} \mathrm{e}$ & $4,64 \mathrm{Ab}$ & $13,04 \mathrm{~B} \mathrm{~b}$ & 19,66 A b \\
\hline TB 02-25 & $3,56 \mathrm{Ae}$ & $4,17 \mathrm{Ab}$ & 11,29 B b & $17,48 \mathrm{AC}$ \\
\hline TB 02-26 & $3,25 \mathrm{~B} \mathrm{e}$ & $4,26 \mathrm{Ab}$ & $12,86 \mathrm{~B} \mathrm{~b}$ & 18,19 A C \\
\hline Vinho 141 & $3,71 \mathrm{Ad}$ & $1,91 \mathrm{Bd}$ & $14,76 \mathrm{~B} \mathrm{a}$ & $20,40 \mathrm{~A} \mathrm{a}$ \\
\hline ZL-1 & $4,95 \mathrm{AC}$ & $4,55 \mathrm{Ab}$ & $12,44 \mathrm{~B} \mathrm{~b}$ & $18,48 \mathrm{~A} \mathrm{~b}$ \\
\hline
\end{tabular}

Médias seguidas por mesma letra maiúscula na linha e minúsculas na coluna não diferem pelo teste de Scott- Knott ao nível de $5 \%$

Em Sobradinho, dez genótipos apresentaram superiores quanto ao teor $\mathrm{Mg}$, destacando-se TB 02-20, AS-7 e TB 02-21, variando entre 0,70 a $0,61 \mathrm{~g} \mathrm{~kg}^{-1}$ (Tabela 3). Os teores mais baixos foram constatados em Preto Ibérico, Carioca, BRS Intrépido, Amarelinho lolanda e ZL-1 variando entre 0,25 e $0,27 \mathrm{~g} \mathrm{~kg}^{-1}$. Nas sementes provenientes de São Luiz Gonzaga, Guabiju Brilhante e Preto Ibérico apresentaram os melhores resultados de 0,34 e $0,28 \mathrm{~g} \mathrm{~kg}^{-1}$, respectivamente, embora não diferindo de outros genótipos, enquanto TB 02-26 e CK-4 apresentaram os teores mais baixos, de $0,15 \mathrm{~g} \mathrm{~kg}$ 1 , em ambos. Os teores encontrados nos dois locais foram inferiores ao observado por Salum et al.(2008), em sementes de feijão cultivadas em Botucatu-SP. 
Tabela 3. Teores de magnésio ( $\mathrm{Mg}$ ) e de cálcio (Ca) em sementes produzidas em Sobradinho e São Luiz Gonzaga, 2012.

\begin{tabular}{|c|c|c|c|c|}
\hline \multirow[t]{2}{*}{ Genótipos } & \multicolumn{2}{|c|}{$\mathrm{Mg}\left(\mathrm{g} \mathrm{kg}^{-1}\right)$} & \multicolumn{2}{|c|}{$\mathrm{Ca}\left(\mathrm{g} \mathrm{kg}^{-1}\right)$} \\
\hline & Sobradinho & SL Gonzaga & Sobradinho & S L Gonzaga \\
\hline AM-10 & $0,30 \mathrm{Af}$ & $0,25 \mathrm{Ab}$ & $1,05 \mathrm{AC}$ & $0,43 \mathrm{~B} \mathrm{a}$ \\
\hline Amarelinho Iolanda & 0,25 Af & $0,19 \mathrm{~B} \mathrm{C}$ & $0,57 \mathrm{Ae}$ & $0,26 \mathrm{~B} \mathrm{~d}$ \\
\hline AS-7 & $0,61 \mathrm{Ab}$ & 0,19 B C & $3,42 \mathrm{~A} \mathrm{a}$ & $0,31 \mathrm{BC}$ \\
\hline BRS Intrépido (T) & 0,26 Af & 0,19 B C & $0,58 \mathrm{Ae}$ & $0,42 \mathrm{~B} \mathrm{a}$ \\
\hline Carioca $(\mathrm{T})$ & 0,26 Af & $0,26 \mathrm{Ab}$ & $1,18 \mathrm{~A} \mathrm{C}$ & $0,32 \mathrm{~B} \mathrm{C}$ \\
\hline CK-4 & $0,32 \mathrm{Ae}$ & $0,15 \mathrm{~B} \mathrm{C}$ & $1,11 \mathrm{Ac}$ & $0,30 \mathrm{~B} \mathrm{C}$ \\
\hline Guabiju Brilhante & $0,51 \mathrm{AC}$ & $0,34 \mathrm{~B} \mathrm{a}$ & 0,42 Af & $0,33 \mathrm{AC}$ \\
\hline Preto Ibérico & $0,25 \mathrm{Af}$ & $0,28 \mathrm{Ab}$ & $0,55 \mathrm{Ae}$ & $0,38 \mathrm{~B} \mathrm{~b}$ \\
\hline TB 02-20 & $0,70 \mathrm{Aa}$ & $0,24 \mathrm{~B} \mathrm{~b}$ & $3,20 \mathrm{Ab}$ & $0,26 \mathrm{~B} \mathrm{~d}$ \\
\hline TB 02-21 & $0,61 \mathrm{Ab}$ & $0,18 \mathrm{~B} \mathrm{C}$ & $3,32 \mathrm{Aa}$ & $0,32 \mathrm{~B} \mathrm{C}$ \\
\hline TB 02-25 & $0,50 \mathrm{AC}$ & $0,20 \mathrm{~B} \mathrm{C}$ & 0,41 Af & $0,31 \mathrm{~B} \mathrm{C}$ \\
\hline ТВ 02-26 & $0,40 \mathrm{Ad}$ & $0,15 \mathrm{~B} \mathrm{C}$ & $0,38 \mathrm{Af}$ & $0,24 \mathrm{Bd}$ \\
\hline Vinho 141 & $0,38 \mathrm{Ad}$ & $0,25 \mathrm{~B} \mathrm{~b}$ & 0,48 Af & $0,34 \mathrm{~B} \mathrm{C}$ \\
\hline ZL-1 & 0,27 Af & $0,23 \mathrm{Ab}$ & $0,90 \mathrm{Ad}$ & $0,37 \mathrm{~B} \mathrm{~b}$ \\
\hline Média & 0,40 & 0,22 & 1,25 & 0,33 \\
\hline CV\% & \multicolumn{2}{|c|}{9,5} & \multicolumn{2}{|c|}{7,3} \\
\hline
\end{tabular}

Médias seguidas por mesma letra maiúscula na linha e minúsculas na coluna não diferem pelo teste de Scott- Knott ao nível de $5 \%$

Os teores de cálcio (Ca) variaram entre 0,38 a $3,42 \mathrm{~g} \mathrm{~kg}^{-1}$, com as sementes produzidas em Sobradinho mostrando resultados superiores aos encontrados por Pereira et al., (2011), que detectou valores entre 0,25 e $0,95 \mathrm{~g} \mathrm{~kg}^{-1}$, em sementes crioulas de feijão, cultivadas em Lages SC. Os teores mais altos desse elemento foram apresentados pelos genótipos AS-7 e TB 02-21 em Sobradinho e AM-10 e BRS Guerreiro em São Luiz Gonzaga, enquanto o valor mais baixo foi observado no genótipo TB 02-26 em ambos os locais.

O genótipo AM-10 apresentou os teores mais alto de ferro com sementes produzidas em São Luiz Gonzaga (Tabela 4). Já com sementes de Sobradinho, o genótipo TB 02-20 apresentou-se com valor mais alto desse nutriente. Os teores mais baixos em Sobradinho foram apresentados pelos genótipos Amarelinho Iolanda, CK-4 e
Guabiju Brilhante, enquanto em São Luiz Gonzaga todos os genótipos foram estatisticamente semelhantes e inferiores ao AM-10. Os teores de ferro em Sobradinho e São Luiz Gonzaga variaram de 0,08 a 0,12 $\mathrm{g} \mathrm{kg}^{-1}$ e 0,06 a 0,10 $\mathrm{g} \mathrm{kg}^{-1}$ respectivamente. Esses valores são similares aos encontrados por Pereira et al. (2011) em genótipos crioulos de feijão em dois anos de cultivo, em Lages - SC, com valores variando entre 0,06 a 0,12 e 0,08 a $0,16 \mathrm{~g} \mathrm{~kg}^{-1}$ respectivamente e aos encontrados por Mesquita et al. (2007) avaliando linhagens melhoradas no Paraná, que detectou valores entre 0,07 a 0,13 g $\mathrm{kg}^{-1}$. Por outro lado, se mostraram superiores aos resultados de trabalhos com cultivares de feijão que variaram de 0,06 a 0,09 $\mathrm{g} \mathrm{kg}^{-1}$ e de acessos provenientes do Centro Internacional de Agricultura Tropical (CIAT) que variaram entre 0,05 a 0,09 $\mathrm{g} \mathrm{kg}^{-1}$ (BEEBE et al., 2000). 
Tabela 4. Teores de ferro (Fe) e de zinco (Zn) em sementes produzidas em Sobradinho e São Luiz Gonzaga, Pelotas 2012.

\begin{tabular}{|c|c|c|c|c|}
\hline \multirow[t]{2}{*}{ Genótipos } & \multicolumn{2}{|c|}{$\mathrm{Fe}\left(\mathrm{g} \mathrm{kg}^{-1}\right)$} & \multicolumn{2}{|c|}{$\mathrm{Zn}\left(\mathrm{mg} \mathrm{kg}^{-1}\right)$} \\
\hline & Sobradinho & SL Gonzaga & Sobradinho & S L Gonzaga \\
\hline AM-10 & $0,11 \mathrm{~A} \mathrm{~b}$ & $0,10 \mathrm{~A} \mathrm{a}$ & 28,27 B C & $32,58 \mathrm{~A} \mathrm{a}$ \\
\hline Amarelinho lolanda & $0,09 \mathrm{Ad}$ & $0,06 \mathrm{~B} \mathrm{~b}$ & $30,57 \mathrm{~A} \mathrm{~b}$ & $24,30 \mathrm{~B} \mathrm{C}$ \\
\hline AS-7 & $0,10 \mathrm{AC}$ & $0,08 \mathrm{~B} \mathrm{~b}$ & 26,27 B C & $29,53 \mathrm{Ab}$ \\
\hline BRS Intrépido (T) & 0,09 A C & 0,07 B b & 29,20 A C & $26,00 \mathrm{~B} \mathrm{C}$ \\
\hline Carioca $(\mathrm{T})$ & $0,09 \mathrm{AC}$ & $0,08 \mathrm{Ab}$ & $30,76 \mathrm{Ab}$ & $26,55 \mathrm{~B} \mathrm{C}$ \\
\hline CK-4 & $0,08 \mathrm{Ad}$ & $0,06 \mathrm{~B} \mathrm{~b}$ & $31,10 \mathrm{Ab}$ & 24,74 B C \\
\hline Guabiju Brilhante & $0,08 \mathrm{Ad}$ & $0,08 \mathrm{Ab}$ & 27,14 A C & $27,45 \mathrm{Ab}$ \\
\hline Preto Ibérico & $0,10 \mathrm{AC}$ & $0,07 \mathrm{~B} \mathrm{~b}$ & $33,30 \mathrm{Ab}$ & 29,33 B b \\
\hline TB 02-20 & $0,12 \mathrm{Aa}$ & 0,07 B b & $31,67 \mathrm{Ab}$ & 26,44 B C \\
\hline TB 02-21 & $0,10 \mathrm{AC}$ & 0,08 B b & $27,97 \mathrm{Ac}$ & $22,57 \mathrm{~B} \mathrm{C}$ \\
\hline TB 02-25 & $0,10 \mathrm{AC}$ & $0,07 \mathrm{~B} \mathrm{~b}$ & $26,00 A C$ & $28,47 \mathrm{Ab}$ \\
\hline TB 02-26 & $0,09 \mathrm{AC}$ & $0,06 \mathrm{~B} \mathrm{~b}$ & $28,75 \mathrm{AC}$ & $28,58 \mathrm{Ab}$ \\
\hline Vinho 141 & $0,10 \mathrm{AC}$ & $0,07 \mathrm{~B} \mathrm{~b}$ & $32,25 \mathrm{~A} \mathrm{~b}$ & $27,23 \mathrm{~B} \mathrm{~b}$ \\
\hline ZL-1 & $0,10 \mathrm{Ac}$ & $0,07 \mathrm{~B} \mathrm{~b}$ & $37,22 \mathrm{Aa}$ & $28,65 \mathrm{~B} \mathrm{~b}$ \\
\hline
\end{tabular}

Médias seguidas por mesma letra maiúscula na linha e minúsculas na coluna não diferem pelo teste de Scott-Knott ao nível de 5\%.

O genótipo ZL-1 apresentou o teor mais alto de $\mathrm{Zn}$, em Sobradinho, assim como AM-10 foi superior em São Luiz Gonzaga. Nas sementes provenientes de Sobradinho, o teor mais baixo de Zn foi observado em TB 02-25. Em São Luiz Gonzaga foi em TB 02-21. Os teores de Zn, nos dois locais, foram idênticos aos encontrados por Salum et al. (2008), que constatou valores entre 30,06 a $28,67 \mathrm{mg} \mathrm{kg}^{-1}$. Tais fatos indicam que o teor deste elemento pode ser relativamente estável em função da época de semeadura e fatores genéticos relacionados.

As variações no teor dos nutrientes associadas às condições ambientais, durante 0 desenvolvimento das plantas e das sementes, foram constatadas por Lemos et al. (2004), que enfatizam, ainda a influência do genótipo sobre aquela característica. Sabe-se que tanto o estresse hídrico como a alta temperatura durante o período de enchimento de grãos podem ser possíveis explicações para as variações na concentração de nutrientes no grão, principalmente o nitrogênio (RANGEL et al., 2007). Pereira et al. (2011), em trabalho avaliando o teor de nutrientes em feijão crioulo, observou redução significativa no acúmulo de alguns nutrientes no ano de cultivo, que apresentou, na fase de florescimento e formação de vagens, alta precipitação, fato que pode ser constatado principalmente em São Luiz Gonzaga, na fase final do ciclo.
Quanto a avaliação dos teores dos diferentes nutrientes observou-se que a maior variação foi apresentada no teor de $P$ onde quatro genótipos foram superiores em Sobradinho, outros seis foram superiores em São Luiz Gonzaga e quatro não diferiram. Para os teores de $\mathrm{Ca}, \mathrm{Mg}$, $\mathrm{Zn}$ e Fe as sementes produzidas em Sobradinho apresentaram o maior número de genótipos com resultados superiores em relação às sementes produzidas em São Luiz Gonzaga. Neste local o maior número de genótipos superiores foi observado para o teor de ferro.

Quanto a avaliação da qualidade fisiológica das sementes, verificou-se que, nas sementes produzidas em Sobradinho, os genótipos AM-10, Amarelinho lolanda, AS-7, BRS Intrépido, Carioca, Preto Ibérico, TB 02-20, TB 0221 e ZL-1 foram aqueles que apresentaram melhores resultados no teste de germinação, enquanto TB 02-26, Vinho 141 e Guabiju Brilhante os que se apresentaram inferiores (Tabela 5). Em São Luiz Gonzaga, TB 02-26, Vinho 141 e Amarelinho Iolanda, apresentaram resultados menos expressivos, enquanto os demais foram estatisticamente iguais. É possível constatar que os genótipos AM-10, AS-7, Preto Ibérico, TB 02-20, TB 02-21 e ZL-1 destacaram-se positivamente nos dois locais de cultivos, assim como as testemunhas BRS Intrépido e Carioca. Os genótipos crioulos e melhorados por seleção participativa apresentaram resultados superiores a $85 \%$, tendo vários deles apresentado 
germinação superior a 95\%. Atualmente o padrão mínimo para comercialização de sementes de feijão no Brasil é de $80 \%$ (BRASIL, 2009).

Tabela 5. Germinação e envelhecimento acelerado (EA) em sementes produzidas em Sobradinho e São Luiz Gonzaga, 2012.

\begin{tabular}{lcccc}
\hline Genótipos & \multicolumn{3}{c}{ Germinação (\%) } & EA (\%) \\
\cline { 2 - 5 } & Sobradinho & SLGonzaga & Sobradinho & SLGonzaga \\
\hline AM-10 & $95 \mathrm{~B} \mathrm{a}$ & $98 \mathrm{~A} \mathrm{a}$ & $93 \mathrm{~B} \mathrm{a}$ & $98 \mathrm{~A} \mathrm{a}$ \\
Amarelinho lolanda & $97 \mathrm{~A} \mathrm{a}$ & $93 \mathrm{~B} \mathrm{~b}$ & $95 \mathrm{~A} \mathrm{a}$ & $97 \mathrm{~A} \mathrm{a}$ \\
AS-7 & $98 \mathrm{~A} \mathrm{a}$ & $97 \mathrm{~A} \mathrm{a}$ & $92 \mathrm{~B} \mathrm{a}$ & $98 \mathrm{~A} \mathrm{a}$ \\
BRS-Guerreiro & $97 \mathrm{~A} \mathrm{a}$ & $98 \mathrm{~A} \mathrm{a}$ & $94 \mathrm{~B} \mathrm{a}$ & $99 \mathrm{~A} \mathrm{a}$ \\
Carioca (T) & $96 \mathrm{~A} \mathrm{a}$ & $98 \mathrm{~A} \mathrm{a}$ & $94 \mathrm{~B} \mathrm{a}$ & $98 \mathrm{~A} \mathrm{a}$ \\
CK-4 & $93 \mathrm{~B} \mathrm{~b}$ & $98 \mathrm{~A} \mathrm{a}$ & $92 \mathrm{~B} \mathrm{a}$ & $98 \mathrm{~A} \mathrm{a}$ \\
Guabiju Brilhante & $88 \mathrm{~B} \mathrm{C}$ & $95 \mathrm{~A} \mathrm{a}$ & $85 \mathrm{~B} \mathrm{~b}$ & $98 \mathrm{~A} \mathrm{a}$ \\
Preto Ibérico & $97 \mathrm{~A} \mathrm{a}$ & $95 \mathrm{~A} \mathrm{a}$ & $95 \mathrm{~A} \mathrm{a}$ & $98 \mathrm{~A} \mathrm{a}$ \\
TB 02-20 & $96 \mathrm{~A} \mathrm{a}$ & $99 \mathrm{~A} \mathrm{a}$ & $93 \mathrm{~A} \mathrm{a}$ & $96 \mathrm{~A} \mathrm{a}$ \\
TB 02-21 & $98 \mathrm{~A} \mathrm{a}$ & $97 \mathrm{~A} \mathrm{a}$ & $92 \mathrm{~A} \mathrm{a}$ & $96 \mathrm{~A} \mathrm{a}$ \\
TB 02-25 & $93 \mathrm{~A} \mathrm{~b}$ & $95 \mathrm{~A} \mathrm{a}$ & $88 \mathrm{~B} \mathrm{~b}$ & $93 \mathrm{~A} \mathrm{a}$ \\
TB 02-26 & $88 \mathrm{~A} \mathrm{C}$ & $85 \mathrm{~A} \mathrm{C}$ & $53 \mathrm{~B} \mathrm{C}$ & $96 \mathrm{~A} \mathrm{a}$ \\
Vinho 141 & $89 \mathrm{~A} \mathrm{C}$ & $92 \mathrm{~A} \mathrm{~b}$ & $88 \mathrm{~B} \mathrm{~b}$ & $97 \mathrm{~A} \mathrm{a}$ \\
ZL-1 & $97 \mathrm{~A} \mathrm{a}$ & $98 \mathrm{~A} \mathrm{a}$ & $96 \mathrm{~A} \mathrm{a}$ & $98 \mathrm{~A} \mathrm{a}$ \\
\hline CV\% & \multicolumn{4}{c}{2,48} \\
\hline
\end{tabular}

Médias seguidas por mesma letra maiúscula na linha e minúsculas na coluna não diferem pelo teste de Scott-Knott ao nível de $5 \%$.

Observando os resultados do teste de envelhecimento acelerado verificou-se o alto potencial fisiológico dos genótipos em ambos locais. Para as sementes produzidas em Sobradinho não houve diferença estatística entre os dez melhores genótipos, no entanto o genótipo TB 02-26 foi inferior aos demais, cujo resultado do alcançou 53\%. Em São Luiz Gonzaga, os genótipos não diferiram estatisticamente. Os bons resultados no teste são superiores aos observados por Teixeira et al. (2005), com sementes crioulas, que observou resultados em torno de $80 \%$.

No teste de frio os genótipos AS-7, BRS Intrépido, Carioca, CK-4, Preto Ibérico, TB 02-20,
TB 02-21 e ZL-1 foram os que se destacaram como os melhores resultados nas sementes produzidas em Sobradinho (Tabela 6). Já os resultados inferiores foram apresentados pelos genótipos TB 02-26 e Guabiju Brilhante. As sementes produzidas em São Luiz Gonzaga não apresentaram diferença estatística entre os genótipos. Assim como ocorreu no teste de germinação, no teste de frio é possível observar maior vigor nos genótipos AS-7, Preto lbérico, TB 02-20, TB 02-21 e ZL-1 juntamente com as testemunhas BRS Intrépido e Carioca. 
Tabela 6. Teste de frio e condutividade elétrica de sementes de feijão produzidas em Sobradinho e São Luiz Gonzaga, safra 2012.

\begin{tabular}{|c|c|c|c|c|}
\hline \multirow[t]{2}{*}{ Genótipos } & \multicolumn{2}{|c|}{ Teste de frio (\%) } & \multicolumn{2}{|c|}{$\mathrm{CE}\left(\mu \mathrm{S} \mathrm{cm}^{-1} 1 \mathrm{~g}^{-1}\right)$} \\
\hline & Sobradinho & S.L.Gonzaga & Sobradinho & S.L.Gonzaga \\
\hline AM-10 & $95 \mathrm{Ab}$ & $96 \mathrm{~A} \mathrm{a}$ & $64,4 \mathrm{Aa}$ & 41,8 B c \\
\hline Amarelinho Iolanda & $93 \mathrm{~B} \mathrm{~b}$ & $97 \mathrm{~A} \mathrm{a}$ & $43,6 \mathrm{Ab}$ & 35,9 A C \\
\hline AS-7 & $98 \mathrm{~A} \mathrm{a}$ & $96 \mathrm{~A} \mathrm{a}$ & $65,9 \mathrm{Aa}$ & $59,5 \mathrm{Aa}$ \\
\hline BRS Guerreiro & $97 \mathrm{~A} \mathrm{a}$ & $97 \mathrm{~A} \mathrm{a}$ & $40,1 \mathrm{Ab}$ & $38,8 \mathrm{AC}$ \\
\hline Carioca $(\mathrm{T})$ & $96 \mathrm{~A} \mathrm{a}$ & $98 \mathrm{~A} \mathrm{a}$ & $67,4 \mathrm{Aa}$ & 52,7 B b \\
\hline CK-4 & $96 \mathrm{~A} \mathrm{a}$ & $97 \mathrm{~A} \mathrm{a}$ & $60,0 \mathrm{Aa}$ & 42,9 B C \\
\hline Guabiju Brilhante & $75 \mathrm{Bd}$ & $97 \mathrm{~A} \mathrm{a}$ & $62,7 \mathrm{Aa}$ & 42,9 B C \\
\hline Preto Ibérico & $97 \mathrm{~A} \mathrm{a}$ & $98 \mathrm{~A} \mathrm{a}$ & $67,8 \mathrm{Aa}$ & $58,4 \mathrm{Aa}$ \\
\hline TB 02-20 & $96 \mathrm{~A} \mathrm{a}$ & $96 \mathrm{~A} \mathrm{a}$ & $74,2 \mathrm{Aa}$ & $64,3 \mathrm{~A} a$ \\
\hline TB 02-21 & $97 \mathrm{~A} \mathrm{a}$ & $97 \mathrm{~A} \mathrm{a}$ & $62,9 \mathrm{Aa}$ & $64,3 \mathrm{Aa}$ \\
\hline TB 02-25 & 89 B C & $96 \mathrm{~A} \mathrm{a}$ & $73,6 \mathrm{Aa}$ & $36,3 \mathrm{~B} \mathrm{C}$ \\
\hline TB 02-26 & $80 \mathrm{~B} \mathrm{~d}$ & $97 \mathrm{~A} \mathrm{a}$ & $59,3 \mathrm{Aa}$ & $37,7 \mathrm{~B} \mathrm{C}$ \\
\hline Vinho 141 & $91 \mathrm{~B} \mathrm{~b}$ & $97 \mathrm{~A} \mathrm{a}$ & $40,2 \mathrm{Ab}$ & $23,3 \mathrm{~B} \mathrm{~d}$ \\
\hline ZL-1 & $97 \mathrm{~A} \mathrm{a}$ & $98 \mathrm{Aa}$ & $67,1 \mathrm{Aa}$ & $52,8 \mathrm{~B} \mathrm{~b}$ \\
\hline Média & 92 & 96 & 60,7 & 46,5 \\
\hline CV\% & \multicolumn{2}{|c|}{1,49} & \multicolumn{2}{|c|}{12,0} \\
\hline
\end{tabular}

Médias seguidas por mesma letra maiúscula na linha e minúsculas na coluna não diferem pelo teste de Scott-Knott ao nível de $5 \%$.

$\begin{aligned} \text { Para os resultados de condutividade } & \text { étrica, em Sobradinho destacaram-se }\end{aligned}$ positivamente Vinho 141, BRS Intrépido e Amarelinho Iolanda, o que é um indicativo da maior integridade das membranas celulares e menor liberação de solutos, além de minimizar o crescimento de microrganismos nocivos à emergência das plântulas (MARCOS FILHO, 2005). Em São Luiz Gonzaga destacou-se positivamente Vinho 141, enquanto TB 02-20, TB 02-21, AS-7 e Preto Ibérico apresentaram os maiores valores de condutividade. Os resultados ficaram entre 23,34 e $74,23 \mu \mathrm{S} . \mathrm{cm}^{-1} 1 . \mathrm{g}^{-1}$, valores esses menores que os encontrados por Coelho (2010) que situaram entre 33,93 e 124,00 $\mu \mathrm{S} . \mathrm{cm}^{-1} 1 \mathrm{~g}^{-1}$. A condutividade constatada em Sobradinho foi $60,67 \mu S . \mathrm{cm}^{-1} 1 . \mathrm{g}^{-1}$, em média, semelhante aos valores constatados por Costa et al. (2008), em sementes com elevado percentual de germinação. Os resultados obtidos não apresentaram boa relação com os resultados dos demais testes de vigor, pois, é esperado que genótipos com alto vigor mostrem baixa condutividade elétrica. Tal fato demonstra que existem outros fatores influenciando o teste.

Em Sobradinho os genótipos AM-10, AS7, Preto Ibérico, TB 02-20, TB 02-21 e ZL-1 mostraram o maior comprimento de raiz, enquanto os menores valores foram apresentados por TB 02-26, Vinho 141, Guabiju Brilhante, Amarelinho lolanda e TB 02-25 (Tabela 7). Em São Luiz Gonzaga os melhores resultados foram obtidos com os genótipos AM-10, AS-7, BRS Intrépido, CK- 4, TB 02-21 e ZL-1, tendo o Amarelinho lolanda, Vinho 141 e TB 02-26 como os menores valores. Os resultados, entretanto, foram superiores aos encontrados por Coelho et. al., (2010) com $11,3 \mathrm{~cm}$ e 2,74 cm, respectivamente, também avaliado em genótipos crioulos. Para Binotti et al. (2008) em cultivares comerciais, os valores situaram em torno de 8 $\mathrm{cm}$. Dessa forma alguns genótipos podem ser considerados potencialmente vigorosos, podendo proporcionar maiores taxas de crescimento no período inicial (DUTRA et al., 2007). 
Tabela 7. Comprimento de raiz e de parte aérea, em $\mathrm{cm}$, de plântulas resultante de sementes produzidas em Sobradinho e São Luiz Gonzaga, 2012.

\begin{tabular}{|c|c|c|c|c|}
\hline \multirow[t]{2}{*}{ Genótipos } & \multicolumn{2}{|c|}{ Comprimento de raiz } & \multicolumn{2}{|c|}{ Comprimento de parte aérea } \\
\hline & Sobradinho & SL Gonzaga & Sobradinho & SL Gonzaga \\
\hline AM-10 & $20,00 \mathrm{~A} \mathrm{a}$ & 11,99 В а & $8,44 \mathrm{Aa}$ & $8,23 \mathrm{~B} \mathrm{a}$ \\
\hline Amarelinho Iolanda & $14,66 \mathrm{Ad}$ & $9,64 \mathrm{~B} \mathrm{C}$ & $4,46 \mathrm{Ae}$ & $2,80 \mathrm{~B} \mathrm{~d}$ \\
\hline AS-7 & $19,84 \mathrm{Aa}$ & $12,31 \mathrm{~B} \mathrm{a}$ & $8,34 \mathrm{Aa}$ & $6,29 \mathrm{Ab}$ \\
\hline BRS-Guerreiro & $17,39 \mathrm{AC}$ & $11,87 \mathrm{~B} \mathrm{a}$ & $6,17 \mathrm{Ad}$ & $6,17 \mathrm{Ab}$ \\
\hline Carioca $(\mathrm{T})$ & $18,91 \mathrm{~A} \mathrm{~b}$ & $11,33 \mathrm{~B} \mathrm{~b}$ & $7,26 \mathrm{AC}$ & $6,20 \mathrm{~B} \mathrm{~b}$ \\
\hline CK-4 & $18,78 \mathrm{Ab}$ & $13,51 \mathrm{~B} \mathrm{a}$ & 6,79 A C & $7,20 \mathrm{~A} \mathrm{a}$ \\
\hline Guabiju Brilhante & $14,92 \mathrm{Ad}$ & $10,98 \mathrm{~B} \mathrm{~b}$ & $4,52 \mathrm{Af}$ & $3,53 \mathrm{BC}$ \\
\hline Preto Ibérico & $20,59 \mathrm{~A} \mathrm{a}$ & $10,80 \mathrm{~B} \mathrm{~b}$ & $8,77 \mathrm{~A} \mathrm{a}$ & $5,86 \mathrm{~B} \mathrm{~b}$ \\
\hline TB 02-20 & $21,60 \mathrm{Aa}$ & $11,29 \mathrm{~B} \mathrm{~b}$ & $7,63 \mathrm{Ab}$ & $6,34 \mathrm{~B} \mathrm{~b}$ \\
\hline TB 02-21 & $19,74 \mathrm{~A} \mathrm{a}$ & $12,08 \mathrm{~B} \mathrm{a}$ & $8,47 \mathrm{~A} \mathrm{a}$ & $7,09 \mathrm{~B} \mathrm{a}$ \\
\hline TB $02-25$ & $14,93 \mathrm{Ad}$ & 10,92 B b & $4,38 \mathrm{Ae}$ & $4,30 \mathrm{AC}$ \\
\hline TB 02-26 & $13,13 \mathrm{~A} \mathrm{e}$ & 9,62 B C & 3,05 Af & $2,96 \mathrm{Ad}$ \\
\hline Vinho 141 & $12,98 \mathrm{~A} \mathrm{e}$ & $8,56 \mathrm{~B} \mathrm{~d}$ & 3,01 Af & $2,24 \mathrm{Ad}$ \\
\hline ZL-1 & 20,30 a a & $12,47 \mathrm{~B} \mathrm{a}$ & $8,17 \mathrm{Aa}$ & $7,39 \mathrm{Aa}$ \\
\hline Média & 17,7 & 11,2 & 6,4 & 5,5 \\
\hline CV\% & \multicolumn{2}{|c|}{5,44} & \multicolumn{2}{|c|}{9,9} \\
\hline
\end{tabular}

Médias seguidas por mesma letra maiúscula na linha e minúsculas na coluna não diferem pelo teste de Scott-Knott ao nível de $5 \%$.

Quanto ao comprimento de parte aérea, em Sobradinho, AM-10, AS-7, Preto Ibérico, TB 02-21 e ZL-1, apresentaram os melhores resultados, enquanto em São Luiz Gonzaga os melhores genótipos foram: AM-10, CK-4, TB 0221 e ZL-1. Os menores resultados de comprimento de parte aérea foram apresentados pelos genótipos TB 02-26 e Vinho 141, nos dois locais de cultivo.

Alguns genótipos que apresentaram os melhores resultados de germinação, envelhecimento acelerado e teste de frio em São Luiz Gonzaga, não mostraram o mesmo comportamento em Sobradinho. Tal fato pode estar relacionado a precipitação maior, observada no final do ciclo em São Luiz Gonzaga. A época de semeadura pode ter influenciado na qualidade das sementes, como foi observado em soja por Nakagawa et al. (1986). Sementes de qualidade, de soja, foram obtidas em colheitas realizadas sob temperatura amena e umidade relativa do ar mais baixa (AGUERO et al., 1997). Esse fato pode ter contribuído para que a qualidade fisiológica das sementes tenha apresentado comportamento superior em São Luiz Gonzaga, cuja temperatura média foi mais amena do que Sobradinho, na fase final de cultivo. A condutividade elétrica apresentou os melhores resultados para as sementes produzidas em São Luiz Gonzaga em oito genótipos, não diferindo para os demais. Para o comprimento de raiz as sementes produzidas em Sobradinho apresentaram os melhores resultados. Já para comprimento de parte aérea metade dos genótipos com sementes produzidas em Sobradinho apresentou os melhores resultados e a outra metade não diferiu das sementes produzidas em São Luiz Gonzaga.

De modo geral os genótipos AM-10, AS-7, Preto Ibérico, TB 02-21 e ZL-1 apresentaram os melhores resultados na maioria dos testes de vigor independente de local de cultivo, evidenciando sua superioridade sobre as testemunhas BRS Intrépido e Carioca. O fato, por exemplo, de apresentar maior comprimento de raiz, pode favorecer a um maior e mais rápido estabelecimento da cultura em campo. Segundo Rosolem e Marubayashi (1994) o feijão é uma planta muito exigente em nutrientes em função do pequeno e pouco profundo sistema radicular e ao seu ciclo curto. Alguns genótipos apresentaram resultados negativos na maioria dos testes como TB 02-26 e Vinho 141, nos dois locais de cultivo. Esses genótipos, ambos de cor, por terem hábito determinado, possivelmente sejam mais suscetíveis a estresses no final do ciclo o que influencia a germinação das sementes.

Pode-se observar na tabela 8 que houve correlação positiva e significativa entre a concentração de cálcio, em Sobradinho, e fosforo e manganês, em São Luiz Gonzaga, com diversos 
testes de vigor. Como houve interação significativa entre ambiente e genótipos, as correlações estão apresentadas separadamente para cada local analisado. Nas sementes produzidas em Sobradinho foram observadas correlações significativas do teor de $\mathrm{Ca}$ na semente e comprimento de raiz e parte aérea das plântulas, e correlação significativa, porém mais discreta, do elemento com a germinação, condutividade elétrica e o teste de frio, assim como Fe e Mn com o teste de frio, comprimento de parte aérea e comprimento de raiz. $\mathrm{O} F$ também apresentou correlação significativa com condutividade elétrica e comprimento de raiz.

O vigor é um conjunto de variáveis extremamente difíceis de sofrer incremento, de modo que quaisquer elementos que o possam alterá-lo positivamente são de extrema importância para o estabelecimento de cultivos, neste sentido (LEMES et al., 2015; SANTOS et al., 2017; TEIXEIRA et al., 2011) observam a dificuldade de que o vigor de sementes seja incrementado e contribua para o estabelecimento dos cultivos.B

Tabela 8 - Coeficientes de correlação entre o teor de nutrientes e os testes de qualidade das sementes produzidas em Sobradinho e São Luiz Gonzaga, RS, 2012.

\begin{tabular}{|c|c|c|c|c|c|c|}
\hline \multirow[b]{3}{*}{ Nutrientes } & \multicolumn{6}{|c|}{ Testes de qualidade das sementes } \\
\hline & \multicolumn{5}{|c|}{ Sobradinho } & \multirow[b]{2}{*}{ CPA } \\
\hline & G & EA & TF & C.E & $\mathrm{CR}$ & \\
\hline Fósforo & $-0,14^{\text {ns }}$ & $0,18^{\text {ns }}$ & $-0,30 *$ & $-0,10^{\text {ns }}$ & $-0,17^{\text {ns }}$ & $-0,12^{\text {ns }}$ \\
\hline Cálcio & $0,36^{*}$ & $0,22^{\mathrm{ns}}$ & $0,32 *$ & $0,32 *$ & $0,54 * *$ & $0,52 * *$ \\
\hline Potássio & $-0,11^{\text {ns }}$ & $0,10^{\mathrm{ns}}$ & $0,11^{\mathrm{ns}}$ & $-0,16^{\text {ns }}$ & $-0.06^{\mathrm{ns}}$ & $-0.06^{\mathrm{ns}}$ \\
\hline Magnésio & $0,17^{\mathrm{ns}}$ & $-0,02^{\text {ns }}$ & $0,14^{\mathrm{ns}}$ & $0,17^{\mathrm{ns}}$ & $0,17^{\mathrm{ns}}$ & $0,11^{\mathrm{ns}}$ \\
\hline Ferro & $0,23^{\mathrm{ns}}$ & $0,21^{\mathrm{ns}}$ & $0,32 *$ & $0,44^{* *}$ & $0,41^{* *}$ & $0,37 *$ \\
\hline Zinco & $0,01^{\mathrm{ns}}$ & $0,16^{\mathrm{ns}}$ & $0,03^{\mathrm{ns}}$ & $0,08^{\mathrm{ns}}$ & $0,16^{\mathrm{ns}}$ & $0,10^{\mathrm{ns}}$ \\
\hline Manganês & $0,21^{\mathrm{ns}}$ & $0,28^{\mathrm{ns}}$ & $0,33^{*}$ & $0,20^{\mathrm{ns}}$ & $0,37^{*}$ & $0.33 *$ \\
\hline \multicolumn{7}{|c|}{ São Luiz Gonzaga } \\
\hline Fósforo & $0,32^{*}$ & $0,05^{\mathrm{ns}}$ & $-0,14^{\mathrm{ns}}$ & $0,60^{* *}$ & $0,42 * *$ & $0,59 * *$ \\
\hline Cálcio & $0,44 * *$ & $0,30^{\text {ns }}$ & $0,15^{\mathrm{ns}}$ & $0,03^{\mathrm{ns}}$ & $0,21^{\text {ns }}$ & $0,30^{\mathrm{ns}}$ \\
\hline Potássio & $0,15^{\mathrm{ns}}$ & $0,22^{\mathrm{ns}}$ & $-0,23^{\mathrm{ns}}$ & $0,31^{*}$ & $0,09^{\text {ns }}$ & $0,37^{*}$ \\
\hline Magnésio & $0,40 * *$ & $0,31^{*}$ & $0,17^{\mathrm{ns}}$ & $-0,06^{\mathrm{ns}}$ & $0,25^{\mathrm{ns}}$ & $0,23^{\mathrm{ns}}$ \\
\hline Ferro & $0,43 * *$ & $0,13^{\mathrm{ns}}$ & $-0,23^{\text {ns }}$ & $0,08^{\mathrm{ns}}$ & $0,38 *$ & $0,39 * *$ \\
\hline Zinco & $0,05^{\mathrm{ns}}$ & $0,13^{\mathrm{ns}}$ & $-0,14^{\mathrm{ns}}$ & $-0,12^{\mathrm{ns}}$ & $0,13^{\text {ns }}$ & $0,13^{\mathrm{ns}}$ \\
\hline Manganês & $0,40 * *$ & $0,55^{* *}$ & $0,25^{\mathrm{ns}}$ & $-0,18^{n s}$ & $0,36 *$ & $0,32 *$ \\
\hline
\end{tabular}

*correlação significativa a $5 \%$ de probabilidade; ** correlação significativa a $1 \%$; G: germinação, TF: teste de frio, EA: envelhecimento acelerado, CE: condutividade elétrica, CR: comprimento de raiz e CPA: comprimento de parte aérea.

Em São Luiz Gonzaga foram observadas correlações altamente significativas e alto coeficiente de correlação (maior de 0,50 ) do teor de $P$ com a condutividade elétrica e o comprimento de parte aérea das plântulas, entretanto também apresentou correlação, porém mais discreta com percentagem de germinação das sementes. Os elementos que exerceram maior influência no vigor das sementes foram $\mathrm{P}, \mathrm{Ca}$, Fe e $\mathrm{Mn}$, demonstrado pelo número de correlações significativas apresentadas. O Ca, além de apresentar correlação com o comprimento de raiz e de parte aérea da plântula, apresentou correlação, porém mais discreta, com a germinação. Adams et al. (1993) determinou que há alta correlação entre a porcentagem de germinação com o teor de $\mathrm{Ca}$ da semente, em amendoim. Da mesma forma outros elementos como, $\mathrm{Mg}$, Fe e $\mathrm{Mn}$ apresentaram correlação discreta com a germinação. Porém o $\mathrm{Mn}$ também apresentou alta correlação com o teste de envelhecimento acelerado. Esse resultado concorda com Sá (1994), o qual coloca a importância do elemento sobre o potencial de armazenamento das sementes.

\section{CONCLUSÕES}

As sementes dos genótipos: AM-10, AS-7, Preto Ibérico, TB 02-21 e ZL-1 são aquelas que apresentam o potencial fisiológico mais elevado, em ambos os ambientes para a maioria dos testes de vigor. 
O teor de nutrientes na semente exerce influência na qualidade das sementes, principalmente fósforo e manganês, em Sobradinho e cálcio, em São Luiz Gonzaga.

\section{REFERÊNCIAS}

ADAMS, J.F.; HARTOZOG. D.L.; NELSON, D.B. Supplemental calcium application on yield, grade, and seed quality of runner peanut. Agronomy Journal, v.85, p.86-93, 1993.

AGUERO, J.A.P.; VIEIRA, R.; BITTENCOURT, S.R.M. Avaliação da qualidade fisiológica em sementes de soja. Revista Brasileira de Sementes, v.19, n.2, p.254-259, 1997.

ANTUNES, I. F.; CHOLLET, C.B.; EMYGDIO, B.M. RODRIGUES, L.S.; MASTRANTONIO, J.J.S.; SILVEIRA, E.P. Vade-mécum das cultivares de feijão no Rio Grande do Sul. Pelotas: Embrapa Clima Temperado, 2007. 59 p. (Embrapa Clima Temperado. Documentos, 193).

BINOTTI, F.F.S.; HAGA, K.I.; CARDOSO, E.D.; ALVES, C. Z.; SÁ, M. E.; ARF, O. Efeito do período de envelhecimento acelerado no teste de condutividade elétrica e na qualidade fisiológica de sementes de feijão. Acta Scientiarum. Agronomy, Maringá, v.30, n.2, p.247-254, 2008.

BRASIL. Ministério da Agricultura, Pecuária e Abastecimento. Regras para análises de sementes. Brasília: Mapa / ACS, 2009. 399p.

COELHO, C.M.M.; MOTTA, M.R; SOUZA, C.A.; MIQUELLUTI, D.J. Potencial fisiológico em sementes de cultivares de feijão crioulo (Phaseolus vugaris L.) Revista Brasileira de Sementes, v. 32, n. 3 p. 097-105, 2010.

DISME/INMET; CPPMet/UFPEL. Boletim climático, novembro-dezembro-janeiro (2011-2012) Estado do Rio Grande do Sul. Ano 09 n.10. Porto Alegre, outubro de 2011. Disponível em: <http://www.inmet.gov.br/portal/arq/clima/prog clima rs/bol out2011.pdf>. Acesso em: 08 abr. 2018.

DISME/INMET; CPPMet/UFPEL. Boletim climático, fevereiro-março-abril (2012) Estado do Rio Grande do Sul. Ano 10 n.01. Pelotas, 24 de janeiro de 2012. Disponível em: < http://www.inmet.gov.br/portal/arq/clima/prog clima rs/bol jan2012.pdf>. Acesso em: 08 abr. 2018.

DUTRA, A.S.; TEÓFILO, E.M.; MEDEIROS FILHO, S.; DIAS, F.T.C. Qualidade fisiológica de sementes de feijão caupi em quatro regiões do estado do Ceará. Revista Brasileira de Sementes, v.29, n.2, p.111-116, 2007.

LEMES, E. S.; ALMEIDA, A. S.; MENEGHELLO, G. E.; TUNES, L. M.; VILLELA, F. A. Germinação e vigor de sementes de abóbora tratadas com tiametoxam. Pesquisa Agropecuária Tropical, Goiânia, v. 45, n. 1, p. 122-127, jan./mar. 2015.

LEMOS, L.B.; OLIVEIRA, R.S.; PALOMINO, E.C.; SILVA, T.R.B. Características agronômicas e tecnológicas de genótipos de feijão do grupo comercial Carioca. Pesquisa Agropecuária Brasileira, v. 39, n. 4, p. 319-326, 2004.

MAEDA, S.; MENDONÇA, A. L. Época de semeadura: a cultura do feijão no Mato Grosso do Sul. Dourados: EMBRAPA, 1990. p. 39-40.

MARCOS FILHO, J. Fisiologia de sementes de plantas cultivadas. Piracicaba: FEALQ, 2005. 495p.

MESQUITA, F. R.; CORRÊA, A. D.; ABREU, C. M. P.; Lima, R.A.Z.; Abreu, A.F.B. Linhagens de feijão (Phaseolus vulgaris L.): composição química e digestibilidade proteica. Ciência e Agrotecnologia, Lavras, v. 31, n. 1, p. 1114-1121, 2007.

PEREIRA, T.; COELHO, C.M.M.; BOGO, A.; GUIDOLIN, A.F.; MIQUELLUTI, D.J. Diversity in common bean landraces from South-Brazil. Acta Botanica Croatica, v.68, n.1, p. 79-92, 2009.

PEREIRA, T.; COELHO, C.M.M.; SANTOS, J. C.P.; BOGO, A.; MIQUELLUTI, D. J. Diversidade no teor de nutrientes em grãos de feijão crioulo no Estado de Santa Catarina. Acta Botanica Croatica, Maringá, v. 33, n. 3, p. 477-485, 2011.

RANGEL, M.A.S.; MINUZZI, A.; BRACCINI, A.L.; SCAPIM, C.A.; CARDOSO, P.C. Efeitos da interação genótipos $\mathrm{x}$ ambientes no rendimento de grãos $\mathrm{e}$ nos teores de proteína de cultivares de soja. Acta Scientiarum Agronomy, v. 29, n. 3, p. 351-354, 2007.

RIBEIRO, N.D.; JOST, E.; CERUTTI, T.; MAZEIRO, S.M.; POERSCH, N.L. Composição de 
microminerais em cultivares de feijão e aplicações para o melhoramento genético. Bragantia, Campinas, v.67, n.2, p.267-273, 2008.

ROSOLEM, C.A.; MARUBAYASHI, O.M. Arquivo do agrônomo no 7; Seja doutor do seu feijoeiro. Piracicaba: POTAFOS, 1994. Disponível em: <http://www.ipni.org.br/ppiweb/brazil.nsf/87cb8 a98bf72572b8525693e0053ea70/d5fbc829a2f54 298832569f8004695c5/\$FILE/Seja\%20Feijoeiro.p df>. Acesso em: 05 dez. 2012.

SÁ, M.E. Importância da adubação na qualidade de sementes. In: SÁ, M.E; BUZZETI, S. (Eds). Importância da adubação na qualidade dos produtos agrícolas. São Paulo: íCONE, 1994. p.6598

SALUM, J. D.; ZUCARELI, C.; GAZOLA, E.; NAKAGAWA, J. Características químicas e fisiológicas de sementes de feijão em função do teor de fósforo na semente e doses de fósforo no solo. Revista Brasileira de Sementes, v. 30, n. 1, p.140-149, 2008.

SANTOS, A. S.; SOUZA, E. M.; FÉBOLI, A.; NOGUEIRA, D. C.; Testes de vigor em sementes de três cultivares de soja. Revista Conexão Eletrônica, Três Lagoas, v.14, n. 1, 2017.

SILVA, F. C. Manual de análises químicas de solos, plantas e fertilizantes. Brasília: Embrapa Solos, Embrapa Informática Agropecuária, 1999. 370 p.

TEIXEIRA, I.R.; BOREM, A.; ARAUJO, G.A.A.; ANDRADE, M.J.B. Teores de nutrientes e qualidade fisiológica de sementes de feijão em resposta à adubação foliar com manganês e zinco. Bragantia, Campinas, v.64, n.1, p.83-88, 2005.

TEIXEIRA, R. N.; TOLEDO, M. Z.; FERREIRA, G.; CAVARIANI, C.; JASPER, S. P.; Germinação e vigor de sementes de crambe sob estresse hídrico. Irriga, Botucatu, v. 16 , n. 1, p. 42-51, jan.-mar. 2011.

Recebido para publicação em 26/04/2017

Revisado em 11/09/2017

Aceito em 10/04/2018 